\title{
Targeting Microbiome: An Alternative Strategy for Fighting SARS-CoV-2 Infection
}

\author{
Ornella Spagnolello ${ }^{a}$ Claudia Pinacchio $^{a}$ Letizia Santinelli $^{\mathrm{a}}$ \\ Paolo Vassalini ${ }^{a}$ Giuseppe Pietro Innocenti ${ }^{a}$ Gabriella De Girolamo ${ }^{a, b}$ \\ Silvia Fabris ${ }^{c}$ Marta Giovanetti $^{c, d, e}$ Silvia Angeletti ${ }^{f}$ Alessandro Russo $^{a, b}$ \\ Claudio M. Mastroiannia, ${ }^{a}$ Massimo Ciccozzic Giancarlo Ceccarellia, b, g \\ Gabriella d'Ettorre ${ }^{a, b}$
}

\begin{abstract}
aDepartment of Public Health and Infectious Diseases, Sapienza University of Rome, Rome, Italy; ${ }^{b}$ COVID-19 Unit, Azienda Policlinico "Umberto I" University Hospital, Rome, Italy; 'Medical Statistic and Molecular Epidemiology Unit, University of Biomedical Campus, Rome, Italy; 'Laboratório de Flavivírus, Instituto Oswaldo Cruz, Fundação Oswaldo Cruz, Rio de Janeiro, Brazil; ' Laboratório de Genética Celular e Molecular, ICB, Universidade Federal de Minas Gerais, Belo Horizonte, Brazil; fUnit of Clinical Laboratory Science, University of Biomedical Campus, Rome, Italy; ${ }^{9}$ Migrant and Global Health Research Organization (Mi-HeRo), Rome, Italy
\end{abstract}

\author{
Keywords \\ Dysbiosis · COVID-19 · Gut-lung axis · Probiotics · Systemic \\ cytokine storm
}

\begin{abstract}
Respiratory and gastrointestinal symptoms are the predominant clinical manifestations of the coronavirus disease 2019 (COVID-19). Infecting intestinal epithelial cells, the severe acute respiratory syndrome coronavirus- 2 may impact on host's microbiota and gut inflammation. It is well established that an imbalanced intestinal microbiome can affect pulmonary function, modulating the host immune response ("gutlung axis"). While effective vaccines and targeted drugs are being tested, alternative pathophysiology-based options to prevent and treat COVID-19 infection must be considered on top of the limited evidence-based therapy currently available. Addressing intestinal dysbiosis with a probiotic supplement may, therefore, be a sensible option to be evaluated, in addition to current best available medical treatments.
\end{abstract}

Herein, we summed up pathophysiologic assumptions and current evidence regarding bacteriotherapy administration in preventing and treating COVID-19 pneumonia.

(c) 2021 S. Karger AG, Basel

\section{Introduction}

The coronavirus disease 2019 (COVID-19), caused by severe acute respiratory syndrome coronavirus-2 (SARSCoV-2), has been more than a disease outbreak. Aside from putting an unprecedented strain on health-care systems, the global economy, and society, it boosted the research community worldwide toward new therapeutic options as never before. Surprisingly, among all treatment strategies tested in Randomized Controlled Studies

Ornella Spagnolello, Claudia Pinacchio and Letizia Santinelli are considered first authors.

Giancarlo Ceccarelli and Gabriella d'Ettorre are last authors. karger@karger.com www.karger.com/che

(c) 2021 S. Karger AG, Basel

Karger ${ }^{\prime}=$
Giancarlo Ceccarelli

Department of Public Health and Infectious Diseases, University of Rome Sapienza Viale del Policlinico 155

IT-00161 Rome (Italy)

giancarlo.ceccarelli@uniroma1.it 
(RCTs) so far, the most effective resulted in being the simplest and mostly available at times [1]. While over the first hit of SARS-CoV-2 outbreak, multiple drugs that had already been tested in other conditions with similar infection patterns (i.e., Ebola virus, MERS-CoV) were deployed at this stage of the pandemic specific methods based on physiopathology to prevent and treat COVID-19 are expected to emerge.

Angiotensin-converting enzyme 2 (ACE2) acts as a functional receptor for SARS-CoV-2 [2]. As the alveolar epithelial cells, enterocytes equally express ACE2 receptors in the brush border membrane [3], representing, therefore, an entry point and reservoir for the virus [4].

A growing body of evidence suggests that alteration of intestinal flora composition, the so-called dysbiosis, which was observed in COVID-19 patients, may play a relevant role in determining the course of the disease by increasing systemic pro-inflammatory cytokine production $[5,6]$. Since oral bacteriotherapy is able to restore the composition of intestinal flora and therefore modulate pro-inflammatory cytokine production [7], its potential clinical impact in COVID-19 patients should be thoroughly evaluated.

\section{SARS-CoV-2 and GUT: An Undervalued Relationship}

In addition to fever and typical pulmonary infection manifestations, an increasing number of patients with COVID-19 reported gastrointestinal symptoms such as diarrhea, anorexia, nausea, vomiting, stomach discomfort, and gastrointestinal bleeding [4, 8-10]. Interestingly, the COVID-19 patients experiencing gastrointestinal symptoms had a more severe respiratory disease to the extent that these symptoms could be used to predict ventilatory support requirement and ICU admission.

It is now well-recognized that ACE2 receptor is equally expressed in the lung and the intestinal epithelium. In particular, studies involving immunofluorescence techniques showed that this protein is largely expressed in gastric, duodenal, and rectal epithelial glandular cells, representing a possible gateway for SARS-CoV-2 [11]. In this context, SARS-CoV-2 could be responsible for gastrointestinal inflammation [12] leading to malabsorption, intestinal disorders, activation of the enteric nervous system, and, ultimately, diarrhea.

Indeed, the interaction of specific SARS-CoV-2 spike proteins with ACE2 receptor could induce pro-inflammatory chemokine and cytokine excessive release. This massive release, a hallmark of COVID-19 patients, leads to an acute intestinal inflammatory response, confirmed by raised levels of fecal calprotectin and serum IL-6, and to multi-organ damage consequent to systemic cytokine storm $[5,6]$.

In addition to that, gut ACE2 is also a relevant regulator of amino acid transport, being a chaperone for the membrane trafficking of neutral amino acid transporter (B0AT1), which is expressed both in the proximal kidney tubule and the small intestine $[13,14]$. Considering that ACE2 deficient mice were found to have low plasma levels of tryptophan, increased susceptibility to ulcerative colitis, and severe diarrhea [15], it was postulated that SARS-CoV-2 might alter intestinal microbiome and inflammatory response affecting local amino acid metabo$\operatorname{lism}[13,15,16]$.

Moreover, this alternative mechanism could also promote an excessive gut permeability through epithelial tight junctions' alterations affecting the intestinal barrier function in COVID-19 patients. This was associated with a profound increase of Zonulin, a well-known physiological regulator of tight junction complex in the digestive tract, which was also found to be correlated with higher mortality in COVID-19 patients [17].

These pieces of evidence point out that the gut may represent a route of infection and a SARS-CoV-2 reservoir $[4,18]$. Indeed, up to $50 \%$ of patients released SARS$\mathrm{CoV}-2$ and its nucleic acid in the stool samples during the disease's acute phase. The infection itself generally lasted longer in COVID-19 patients who had previously experienced gastrointestinal symptoms.

\section{Microbiome and Gut-Lung Axis}

Nobel et al. [19] reported that SARS-CoV-2 infection generally lasted longer in COVID-19 patients who had previously experienced gastrointestinal problems. Besides, subjects with diabetes mellitus, hypertension, cerebrovascular disease, and chronic obstructive pulmonary disease experience a more severe course of COVID-19 disease. All these co-morbidities have a common denominator: gut dysbiosis.

Not surprisingly, compared to healthy controls, some COVID-19 patients showed microbial dysbiosis with decreased levels of beneficial bacteria as Lactobacillus and Bifidobacterium, lower bacterial diversity, and higher relative abundance of opportunistic pathogens including Streptococcus spp., Rothia spp., Veillonella spp., and Actinomyces spp [18]. It is becoming increasingly clear that the loss of certain intestinal bacterial strains might be re- 
sponsible for a dysregulated immune response to SARSCoV-2 [20]. Far away from being considered just part of the digestive tract, the gut is crucially involved in the immune system, hosting a large microbial population that can affect the body's respiratory tract through immune regulation $[21,22]$.

The "gut-lung axis" refers to the bidirectional crosstalk between the intestinal tract and the lungs, whose ultimate scope is to modulate the immune response in both compartments as a result of their respective microbial composition and related patterns [23]. This gut-lung interplay embraces multiple anatomical communications and complex pathways [24]. The mesenteric lymphatic system is one of those. Using this route, intact bacteria, their fragments, or metabolites can cross the intestinal barrier and reach the circulatory system influencing other organs' immune response, among these the lung [2527]. However, although the gut's impact on respiratory function is well reckoned, the available evidence of the other way round is still sparse. Nevertheless, postulated apoptosis dysfunction in the intestine tract related to concurrent respiratory infections [28] may account for COVID-19-associated gastrointestinal symptoms.

On the contrary, some COVID-19 patients who resolved SARS-CoV-2 infection in their respiratory tract exhibited the presence of SARS-CoV-2 RNA in their fecal samples, suggesting that the virus replication in the gastrointestinal tract may be independent of the respiratory compartment $[11,29,30]$. This hypothesis is endorsed by recent studies suggesting that gut involvement in COVID-19 is even more severe and prolonged compared with the respiratory tract [31].

Gut microbiome components have significant microbial inhibitory properties toward lung tissues, accomplished through alveolar macrophage, neutrophils, and natural killer cell activity [32, 33]. To a greater extent, bacterial metabolites, as short-chain fatty acids (SCFAs), are proved to act in the lungs attenuating inflammatory responses. Moreover, some bacterial strains could enhance the release of molecules with antiviral activity like the nuclear factor erythroid 2p45-related factor 2 (Nrf2) and its target Heme oxygenase-1 (HO-1) [34-38].

Although the evidence in COVID-19 is still sparse, bacteriotherapy could represent a potential strategy to counteract SARS-CoV-2 infection. Given the microbiome's crucial role in modulating host immune and inflammatory response, bacteriotherapy may minimize gastrointestinal symptoms and shield the respiratory tract.

\section{Targeting Microbiome to Prevent SARS-CoV-2}

As stated by the WHO, probiotics are "live microorganisms which, when administered in adequate amounts, confer a health benefit on the host" [39]. Historically, the concept of probiotics began around 1,900 by the Nobel laureate Elie Metchnikoff, who discovered that the consumption of live bacteria (Lactobacillus bulgaricus) in yogurt or fermented milk improved some biological features of the gastrointestinal tract [40]. Probiotics are now widely available, generally in dairy products, such as yogurt, dessert, ice cream, juices, and capsules, drops, sachets, etc. The most common strains commercially available belong to the Lactobacillus and Bifidobacterium species, which proved some beneficial effects to the human body when administered in adequate amounts. These mentioned bacterial species are known to be involved in some essential physiological functions such as stimulation of immune response, prevention of pathogenic and opportunistic microbial colonization, production of SCFA, catabolism of carcinogenic substances, and synthesis of vitamins such as B and K [41-43]. In this regard, medical data showed that particular strains of probiotics facilitate the prevention of viral and bacterial infections (such as sepsis, gastroenteritis, and respiratory tract syndrome [4]), improve the intestinal epithelial barrier function, and compete with disease-causing agents for nutrients. Similar to other SARS coronaviruses, SARS-CoV-2 interacts with ACE2 receptor for gut and lung intracellular invasion [2, 24] (Fig. 1).For these reasons, some researchers suggested that ACE inhibitors might benefit patients with COVID-19 by reducing pulmonary inflammation [44] although others argued that ACE inhibitors might enhance viral entry regulating ACE2 levels.

The potential interaction between probiotics and $\mathrm{ACE}$ enzymes was suggested in the previous studies addressing the probiotics' potential antihypertensive effect [45]. Indeed, during food fermentation, probiotics release bioactive peptides able to inhibit the ACE enzymes by blocking the active sites $[46,47]$. The debris of the dead probiotic cells also acted as ACE inhibitors [48].

In this respect, Anwar et al. [49] demonstrated in a computational docking study that 3 metabolites of Lactobacillus plantarum (Plantaricin W, Plantaricin JLA-9, and Plantaricin D) prevent the binding of SARS-CoV-2 with ACE2 receptors suggesting therefore antiviral property of L. plantarum against SARS-CoV-2. Taken all together, these findings stress the assumption that probiotics could compete with ACE2 receptors paving the way for their potential use to prevent SARS-CoV-2 infection.
Spagnolello et al. 


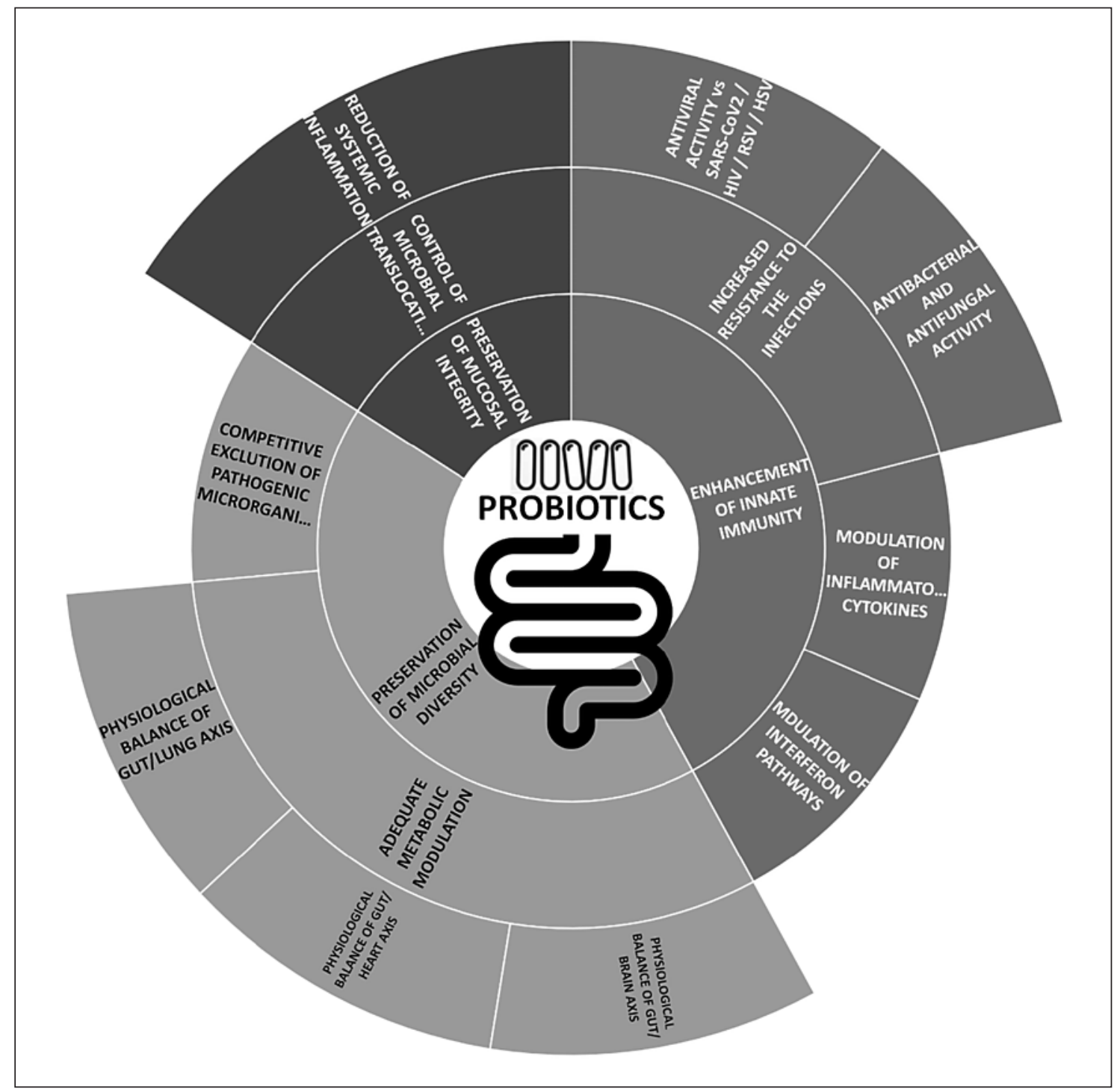

Fig. 1. Mechanisms of action of probiotic supplementation. Legend: SARS-CoV-2, severe acute respiratory syndrome coronavirus-2.

Bacteriotherapy was found to reduce both upper and lower respiratory tract infections [50]. Probiotic lactic acid bacteria were administered directly in the respiratory tract or as oral supplements to improve the immune response and ht viral infections [51]. As an alternative mechanism of action, probiotics were also found to inhibit viruses by interacting directly with them with a mechanism similar to phagocytosis.

More recently, lactobacilli isolated from healthy human noses showed probiotic effects in the form of nasal spray [52], by avoiding the attack of viral particles to mu- cosal cells. These findings also open the chance to deploy probiotics in a nasal spray to boost the immune system and avoid respiratory tract infections .

To the best of our knowledge, no clinical trial has formally investigated the role of probiotics in preventing COVID-19 so far. However, a multicentric RCT is currently evaluating the effects of a 2-month probiotic supplement on the incidence and severity of COVID-19 among health-care workers exposed to SARS-CoV-2 (Table 1) [53]. The trial was completed in October 2020, and results are expected soon. 


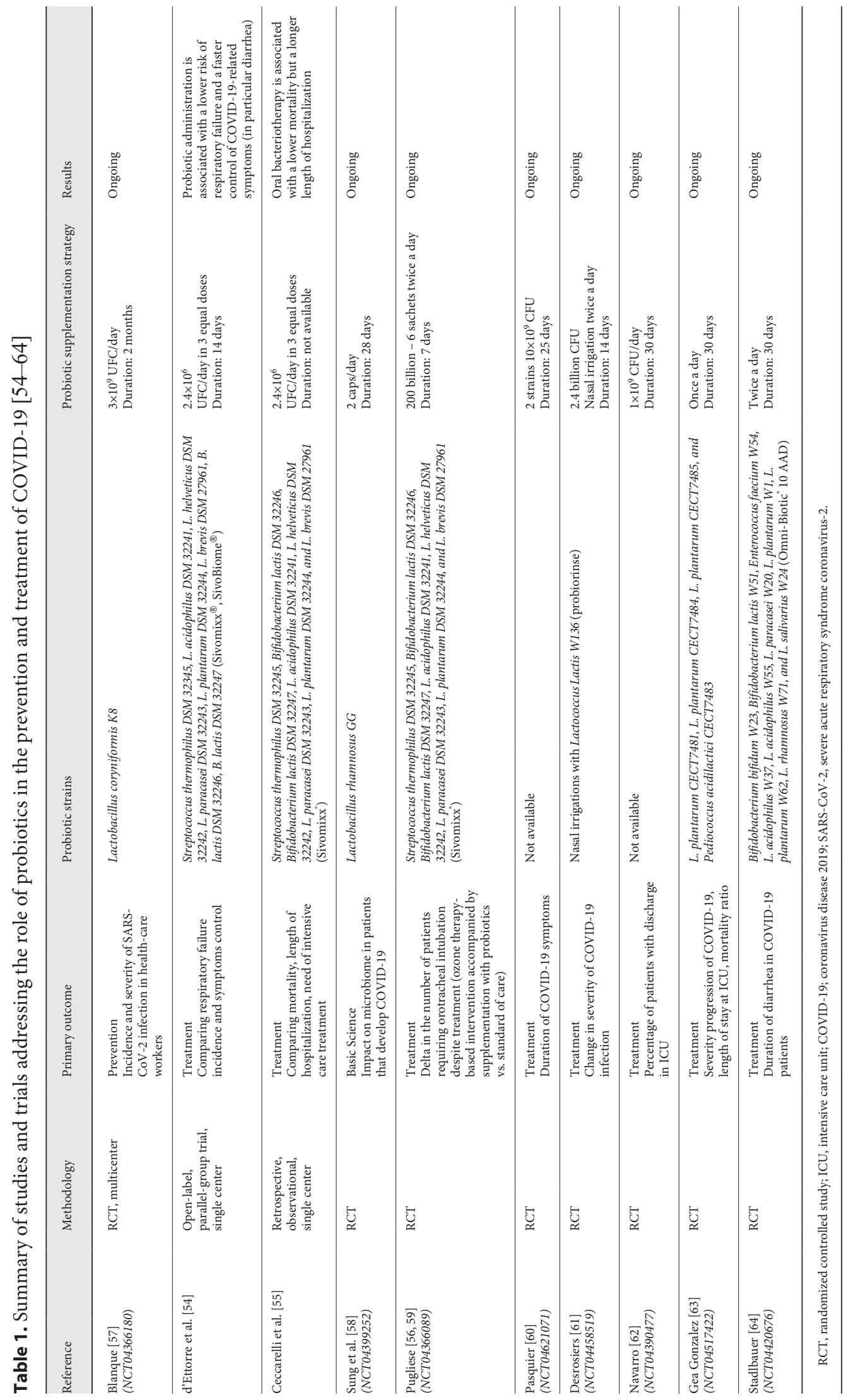


In summary, probiotics may act as antiviral agents interfering with viral entry into cells and/or inhibiting virus replication. This may lead to a limitation in the spread of SARS-CoV-2 in the gut and respiratory tract, as a result of the restoration of the gut and respiratory microbiota.

\section{Targeting Microbiome to ht SARS-CoV2}

Since the gut microbiome is altered in COVID-19 patients [18], probiotics supplementation may help restore the gut microbiota and, therefore, maintain a healthy gutlung axis and minimize translocation of pathogenic bacteria across the gut barrier as well as the chances of secondary bacterial infections. As cytokines storm occurs in patients with severe COVID-19, immune-modulatory effects of probiotics might be relevant to prevent acute lung injury, acute respiratory distress syndrome, and multiple organ failure, which are life-threatening complications of COVID-19 [54].

Boosting immune responses during the incubation and nonsevere stages of COVID-19 infection are perceived as crucial to eliminate the virus and prevent disease progression to severe stages. Administration of certain Bifidobacteria or Lactobacilli has a beneficial impact on influenza virus clearance from the respiratory tract [65]. Besides, probiotic strains increase the levels of type I interferons, the number and activity of antigen-presenting cells, NK cells, T cells, and the levels of systemic and mucosal-specific antibodies in the lungs [65].

Another relevant effect of probiotics is to enforce and maintain the integrity of tight junctions between enterocytes: Hummel et al. [66] showed that gram-positive probiotic lactobacilli modulate epithelial barrier function via their effect on adherence junction protein expression and complex formation [66]. Also, incubation with lactobacilli differentially influences the phosphorylation of adherence junction proteins and the abundance of protein kinase C (PKC) isoforms such as PKC $\delta$ that positively modulates epithelial barrier function.

To this extent, in our previous work, HIV-1-infected patients receiving oral bacteriotherapy exhibited histomorphological and ultrastructural changes in their gut mucosa, characterized by an improvement of epithelial integrity, a reduction of inflammatory infiltrate and enterocyte apoptosis in the terminal ileum, cecum, ascending, transverse, and descending colon [67].

Consistent with previous findings, these immunomodulatory benefits seem to be equally crucial in COVID-19 patients. Based on these shreds of evidence, our group ad- dressed the topic over the past year, carrying out 2 retrospective observational studies including adults with severe COVID-19 pneumonia to investigate the role of oral bacteriotherapy on the top of best available therapy $[54,55]$.

In the first piece of work, we compared respiratory failure incidence and symptoms control in patients with COVID-19 pneumonia receiving a probiotic multistrain formulation $\left(\right.$ Sivomixx ${ }^{\circledR}$, SivoBiome $^{\circledR}$ ) in adjunction to standard medical therapy [54]. Out of 70 patients enrolled in the study, 28 received oral bacteriotherapy for 14 days. According to our results, $92.9 \%$ of the intervention group achieved diarrhea and other symptoms control within $72 \mathrm{~h}$ from study inclusion (vs. less than half in 7 days in the not supplemented group). Moreover, the risk of developing respiratory failure was 8 -fold lower in patients receiving oral bacteriotherapy. Both the prevalence of ICU admission and mortality were lower in the intervention group [54].

In the second study, we extended our sample size. We focused our observation on mortality, ICU admission, and length of hospital staying of patients with COVID-19 pneumonia receiving probiotics as complementary therapy [55]. Out of 200 patients enrolled, 88 received oral bacteriotherapy (Sivomixx ${ }^{\circledR}$ ). Results concerning mortality were quite encouraging: we found a significant reduction in the intervention group ( 11 vs. $30 \%$; $p<0.001$ ). In addition to that, by multivariate analysis, bacteriotherapy emerged as an independent variable associated with a reduced risk for death. In terms of ICU admission, no significant difference among the 2 groups was found. By contrast, we found a longer length of hospitalization in patients receiving bacteriotherapy. We interpreted this data in line with the lower mortality rate of this group [55].

Interestingly, no adverse reactions in patients treated with oral bacteriotherapy were recorded in both studies. In conclusion, for the first time, we determined the role of probiotics in treating patients with COVID-19 pneumonia, providing positive evidence in favor of their implementation in addition to the best available therapy. To the best of our knowledge, 7 RCTs that may soon replicate this insight are currently ongoing (Table 1).

\section{The New Frontier: Are Distinct Microbiome Patterns Associated with Different Risks of COVID-19 Progression?}

Several studies showed that dietary habits and the amount of food consumed could shape human microbiome [68]. Diet in developing countries usually con- 
sists of food containing more fiber than a modern Western diet consisting of food often processed and kept in cold storage [69]. It is now well reckoned that different populations with different diets could have distinct microbiome patterns. Generally, Firmicutes are dominant in people with animal-based diets, whereas Bacteroidetes are dominant in people with a vegetarian diet [70]. Similarly, molecules involved in the digestion of fiber and concentration of SCFAs are differently represented in the microbiome of populations with different diets [71, 72]. Interestingly, a plant food-based diet maintains a more stable microbiota diversity and eubiosis and promotes the microbes that ensue anti-inflammatory response [73]. Preliminary reports suggested that different progression rates to severe disease and fatality rates observed for SARS-CoV-2 in diverse populations could be related to distinct microbiome patterns. For example, it was observed that India reported a fatality rate caused by SARS-CoV-2 lower if compared to other regions consuming meat-rich diet and saturated fatty acids, such as the USA, Brazil, and European countries [74]. Currently, it is not possible to strongly support this hypothesis, but nevertheless, it could be a clue to understand the variations observed in the impact of COVID-19 in populations residing in different geographical areas.

\section{Expert Opinion}

Probiotics may play a beneficial role even though there is much to be discovered on their specific mechanisms of action against SARS-CoV-2. The damage of the gut barrier integrity associated with microbial translocation along with the dysregulated inflammatory response explains why probiotics could represent a valuable therapeutic tool in COVID-19 patients [56, 69, 75-79].

However, clinicians should be mindful that probiotics' clinical benefit depends upon several factors, such as the bacterial composition of different commercial products, manufacturing processes, dose regimen, etc. Several studies had addressed the impact of probiotics in treating many gastrointestinal disorders, such as Clostridium difficile colitis, inflammatory bowel disease, Helicobacter $p y$ lori infection, etc. [70, 71]. Moreover, in recent studies, probiotics were found to restore gut barrier integrity and, therefore, the gut-brain axis in HIV patients [72]. In conclusion, further understanding of gut microbiome modulation on host health is expected to expand probiotic clinical applications soon.

\section{Conclusion}

The "gut-lung axis" pathophysiology suggests that the intestinal microbiota may play a role in counteracting the "cytokine storm," which is now clearly being the cornerstone of COVID-19 disease [80]. Even though evidence coming from clinical trials is still on the way, we showed for the first time a consistent reduction in mortality and more successful symptoms control in patients with COVID-19 pneumonia receiving oral bacteriotherapy as a complementary therapy.

Therefore, we suggest physicians consider the early administration of oral bacteriotherapy on the top of best available treatment while dealing with patients with $\mathrm{CO}$ VID-19 pneumonia, especially in those experiencing gastrointestinal symptoms. This alternative option has multiple advantages, indeed: it is mostly freely available, cheap, and with limited/no adverse effects.

\section{Statement of Ethics}

Not applicable

\section{Conflict of Interest Statement}

All the authors declare no competing interests.

\section{Funding Sources}

The authors did not receive any financial support (funding, grants, and sponsorship) to be acknowledged.

\section{Author Contributions}

All the authors equally contributed to the literature search and manuscript drafting.
References
1 The WHO Rapid Evidence Appraisal for CO- VID-19 Therapies (REACT) Working Group; Sterne JAC, Murthy S, Diaz JV, Slutsky AS, Villar J, et al. Association between adminis- tration of systemic corticosteroids and mor- tality among critically Ill patients with COV- ID-19: a meta-analysis. JAMA. 2020;324(13): 1330-41.
2 Hoffmann M, Kleine-Weber H, Schroeder S, Krüger N, Herrler T, Erichsen S, et al. SARS- CoV-2 cell entry depends on ACE2 and TM- PRSS2 and is blocked by a clinically proven protease inhibitor. Cell. 2020;181(2):271-e8.

Spagnolello et al. 
3 Dhar D, Mohanty A. Gut microbiota and Covid-19- possible link and implications. Virus Res. 2020;285:198018.

4 Lin L, Jiang X, Zhang Z, Huang S, Zhang Z, Fang Z, et al. Gastrointestinal symptoms of 95 cases with SARS-CoV-2 infection. Gut. 2020; 69(6):997-1001.

5 Effenberger M, Grabherr F, Mayr L, Schwaerzler J, Nairz M, Seifert M, et al. Faecal calprotectin indicates intestinal inflammation in COVID-19. Gut. 2020 Aug;69(8):1543-4.

6 Zhang H, Kang Z, Gong H, Xu D, Wang J, Li $Z$, et al. Digestive system is a potential route of COVID-19: an analysis of single-cell coexpression pattern of key proteins in viral entry process. Gut. 2020;69:1010-8.

7 Azad MAK, Sarker M, Wan D. Immunomodulatory effects of probiotics on cytokine profiles. Biomed Res Int. 2018;23:8063647. PMID: 30426014; PMCID: PMC6218795.

8 Cholankeril G, Podboy A, Aivaliotis VI, Tarlow B, Pham EA, Spencer S, et al. High prevalence of concurrent gastrointestinal manifestations in patients with SARS-CoV-2: early experience from California. Gastroenterology. 2020 Aug;159(2):775-7. S0016-5085(20) 30471-6.

9 Goyal P, Choi JJ, Pinheiro LC, Schenck EJ, Chen R, Jabri A, et al. Clinical characteristics of Covid-19 in New York city. N Engl J Med. 2020 Jun 11;382(24):2372-4. NEJMc2010419.

10 Guan WJ, Ni ZY, Hu Y, Liang WH, Ou CQ, $\mathrm{He} \mathrm{JX}$, et al. Clinical characteristics of coronavirus disease 2019 in China. N Engl J Med. 2020;382:1708-20.

11 Xiao F, Tang M, Zheng X, Liu Y, Li X, Shan H. Evidence for gastrointestinal infection of SARS-CoV-2. Gastroenterology. 2020;158(6): 1831-e3.

12 Sanz Segura P, Arguedas Lázaro Y, Mostacero Tapia S, Cabrera Chaves T, Sebastián Domingo JJ. Involvement of the digestive system in covid-19. A review. Gastroenterol Hepatol. 2020;43(8):464-71.

13 Perlot T, Penninger JM. ACE2: from the renin-angiotensin system to gut microbiota and malnutrition. Microbes Infect. 2013;15(13): 866-73.

14 Camargo SM, Singer D, Makrides V, Huggel $\mathrm{K}$, Pos KM, Wagner CA, et al. Tissue-specific amino acid transporter partners ACE2 and collectrin differentially interact with hartnup mutations. Gastroenterology. 2009;136(3): 872-82.

15 Hashimoto T, Perlot T, Rehman A, Trichereau J, Ishiguro $\mathrm{H}$, Paolino $\mathrm{M}$, et al. ACE2 links amino acid malnutrition to microbial ecology and intestinal inflammation. Nature. 2012; 487(7408):477-81.

16 Ceccarelli G, Scagnolari C, Pugliese F, Mastroianni CM, d'Ettorre G. Probiotics and COVID-19. Lancet Gastroenterol Hepatol. 2020 Aug;5(8):721-2. Published online 2020 Jul 13.

17 Giron LB, Dweep H, Yin X, Wang H, Damra M, Goldman AR, et al. Severe COVID-19 is fueled by disrupted gut barrier integrity. medRxiv.
18 Baud D, Dimopoulou Agri V, Gibson GR, Reid G, Giannoni E. Using probiotics to flatten the curve of coronavirus disease COVID-2019 pandemic. Front Public Health. 2020;8:186.

19 Nobel YR, Phipps M, Zucker J, Lebwohl B, Wang TC, Sobieszczyk ME, et al. Gastrointestinal symptoms and COVID-19: case-control study from the United States. Gastroenterology. 2020 Jul;159(1):373-5.e2.

20 Pouya F, Imani Saber Z, Kerachian MA. Molecular aspects of co-morbidities in COVID-19 infection. Arch Bone Jt Surg. 2020 Apr; 8(Suppl 1):226-30. PMID: 32607393; PMCID: PMC7296607.

21 He Y, Wen Q, Yao F, Xu D, Huang Y, Wang J. Gut-lung axis: the microbial contributions and clinical implications. Crit Rev Microbiol. 2017;43(1):81-95.

22 Shukla SD, Budden KF, Neal R, Hansbro PM. Microbiome effects on immunity, health and disease in the lung. Clin Transl Immunol. 2017;6(3):e133.

23 Tulic MK, Piche T, Verhasselt V. Lunggut cross-talk: evidence, mechanisms and implications for the mucosal inflammatory diseases. Clin Exp Allergy. 2016;46:519-28.

24 Li N, Ma WT, Pang M, Fan QL, Hua JL. The commensal microbiota and viral infection: a comprehensive review. Front Immunol. 2019; 10:1551.

25 Bingula R, Filaire M, Radosevic-Robin N, Bey $\mathrm{M}$, Berthon JY, Bernalier-Donadille A, et al. Desired turbulence? Gut-lung axis, immunity, and lung cancer. J Oncol. 2017;2017: 5035371.

26 McAleer JP, Kolls JK. Contributions of the intestinal microbiome in lung immunity. Eur J Immunol. 2018;48(1):39-49.

27 Trompette A, Gollwitzer ES, Yadava K, Sichelstiel AK, Sprenger N, Ngom-Bru C, et al. Gut microbiota metabolism of dietary fiber influences allergic airway disease and hematopoiesis. Nat Med. 2014;20(2):159-66.

28 Perrone EE, Jung E, Breed E, Dominguez JA, Liang Z, Clark AT, et al. Mechanisms of methicillin-resistant staphylococcus aureus pneumonia-induced intestinal epithelial apoptosis. Shock. 2012 Jul;38(1):68-75.

29 Kopel J, Perisetti A, Gajendran M, Boregowda $\mathrm{U}$, Goyal H. Clinical insights into the gastrointestinal manifestations of COVID-19. Dig Dis Sci. 2020;65(7):1932-9.

$30 \mathrm{Wu}$ Y, Guo C, Tang L, Hong Z, Zhou J, Dong $\mathrm{X}$, et al. Prolonged presence of SARS-CoV-2 viral RNA in faecal samples. Lancet Gastroenterol Hepatol. 2020;5(5):434-5.

31 Xu Z, Shi L, Wang Y, Zhang J, Huang L, Zhang $\mathrm{C}$, et al. Pathological findings of COVID-19 associated with acute respiratory distress syndrome. Lancet Respir Med. 2020; 8(4):420-2. HTTPS.
32 Vieira AT, Rocha VM, Tavares L, Garcia CC, Teixeira MM, Oliveira SC, et al. Control of Klebsiella pneumoniae pulmonar y infection and immunomodulation by oral treatment with the commensal probiotic Bifidobacterium longum 5(1A). Microbes Infect. 2016;18: 180-9.

33 Belkacem N, Serafini N, Wheeler R, Derrien M, Boucinha L, Couesnon A, et al. Lactobacillus paracasei feeding improves immune control of influenza infection in mice. PLoS One. 2017;12(9):e0184976.

34 Devadas K, Dhawan S. Hemin activation ameliorates HIV-1 infection via heme oxygenase-1 induction. J Immunol. 2006;176(7): $4252-7$.

35 Hashiba T, Suzuki M, Nagashima Y, Suzuki S, Inoue $\mathrm{S}$, Tsuburai $\mathrm{T}$, et al. Adenovirus-mediated transfer of heme oxygenase- 1 cDNA attenuates severe lung injury induced by the influenza virus in mice. Gene Ther. 2001;8(19): 1499-507.

36 Espinoza JA, León MA, Céspedes PF, Gómez RS, Canedo-Marroquín G, Riquelme SA, et al. Heme oxygenase-1 modulates human respiratory syncytial virus replication and lung pathogenesis during infection. J Immunol. 2017;199(1):212-23.

37 Tseng CK, Lin CK, Wu YH, Chen YH, Chen WC, Young KC, et al. Human heme oxygenase 1 is a potential host cell factor against dengue virus replication. Sci Rep. 2016;6:32176.

38 Hill-Batorski L, Halfmann P, Neumann G, Kawaoka Y. The cytoprotective enzyme heme oxygenase-1 suppresses Ebola virus replication. J Virol. 2013;87(24):13795-802.

39 Joint FAO/WHO Working Group Report on Drafting Guidelines. Guidelines for the evaluation of probiotics in food. London, ON; 2002. p. 1.

40 Benton D, Williams C, Brown A. Impact of consuming a milk drink containing a probiotic on mood and cognition. Eur J Clin Nutr. 2007 Mar;61(3):355-61. Epub 2006 Dec 6. PMID: 17151594.

41 Logan AC, Venket Rao A, Irani D. Chronic fatigue syndrome: lactic acid bacteria may be of therapeutic value. Med Hypotheses. 2003; 60(6):915-23.

42 Williams B, Landay A, Presti RM. Microbiome alterations in HIV infection a review. Cell Microbiol. 2016;18(5):645-51.

43 Ceccarelli G, Statzu M, Santinelli L, Pinacchio C, Bitossi C, Cavallari EN, et al. Challenges in the management of HIV infection: update on the role of probiotic supplementation as a possible complementary therapeutic strategy for cART treated people living with HIV/ AIDS. Expert Opin Biol Ther. 2019 Sep;19(9): 949-65. Epub 2019 Jul 10. PMID: 31260331.

44 Meng J, Xiao G, Zhang J, He X, Ou M, Bi J, et al. Renin-angiotensin system inhibitors improve the clinical outcomes of COVID-19 patients with hypertension. Emerg Microbes Infect. 2020;9(1):757-60 
45 Robles-Vera I, Toral M, Romero M, Jiménez R, Sánchez M, Pérez-Vizcaíno F, et al. Antihypertensive effects of probiotics. Curr Hypertens Rep. 2017 Apr;19(4):26.

46 Ayyash MM, Sherkat F, Shah NP. The effect of $\mathrm{NaCl}$ substitution with $\mathrm{KCl}$ on Akawi cheese: chemical composition, proteolysis, angiotensin-converting enzyme-inhibitory activity, probiotic survival, texture profile, and sensory properties. J Dairy Sci. 2012; 95(9):4747-59.

47 Ayyash M, Olaimat A, Al-Nabulsi A, Liu SQ. Bioactive properties of novel probiotic lactococcus lactis fermented camel sausages: cytotoxicity, angiotensin-converting enzyme inhibition, antioxidant capacity, and antidiabetic activity. Food Sci Anim Resour. 2020;40: $155-71$.

48 Miremadi F, Ayyash M, Sherkat F, Stojanovska L. Cholesterol reduction mechanisms and fatty acid composition of cellular membranes of probiotic lactobacilli and bifidobacteria. J Funct Foods. 2014;9:295-305.

49 Anwar F, Altayb HN, Al-Abbasi FA, Al-Malki AL, Kamal MA, Kumar V. Antiviral effects of probiotic metabolites on COVID-19. J Biomol Struct Dyn. 2020:1-10.

50 Campbell K. How some probiotic scientists are working to address COVID-19. 2020. Available from: https://isappscience.org/tag/ covid-19/ Accessed 2020 May 04.

51 Barbieri N, Herrera M, Salva S, Villena J, Alvarez S. Lactobacillus rhamnosus CRL1505 nasal administration improves recovery of $\mathrm{T}$ cell mediated immunity against pneumococcal infection. 2017.

52 De Boeck I, van den Broek MFL, Allonsius CN, Martens K, Wuyts S, Wittouck S, et al. Lactobacilli have a niche in the human nose. SSRN Electron J. 2019;31:107674.

53 Evaluation of the probiotic lactobacillus coryniformis K8 on COVID-19 prevention in healthcare workers. ClinicalTrials.gov Identifier: NCT04366180 [last access 4 Jan 2021]. Available at: https://clinicaltrials.gov/ct2/ show/NCT04366180.

54 d'Ettorre G, Ceccarelli G, Marazzato M, Campagna $G$, Pinacchio $C$, Alessandri $F$, et al. Challenges in the management of SARSCoV2 infection: the role of oral bacteriotherapy as complementary therapeutic strategy to avoid the progression of COVID-19. Front Med. 2020;7:389.

55 Ceccarelli G, Borrazzo C, Pinacchio C, Santinelli L, Innocenti GP, Cavallari EN, et al. Oral bacteriotherapy in patients with COVID-19: a Retrospective Cohort Study. Front Nutr. 2020;7:613928
56 Araimo F, Imperiale C, Tordiglione P, Ceccarelli G, Borrazzo C, Alessandri F, et al. Ozone as adjuvant support in the treatment of COVID-19: a preliminary report of probiozovid trial. J Med Virol. 2020 Oct 28.

57 Blanque RR. Evaluation of the Probiotic Lactobacillus Coryniformis K8 on COVID-19 Prevention in Healthcare Workers [last access 18 March 2021]. Available at: https://clinicaltrials.gov/ct2/show/NCT04366180.

58 Sung A, Wischmeyer P. Effect of Lactobacillus on the Microbiome of Household Contacts Exposed to COVID-19 [last access 18 March 2021]. Available at: https://clinicaltrials.gov/ct2/show/NCT04399252.

59 Pugliese F. Oxygen-Ozone as Adjuvant Treatment in Early Control of COVID-19 Progression and Modulation of the Gut Microbial Flora (PROBIOZOVID) [last access 18 March 2021]. Available at: https://clinicaltrials.gov/ ct2/show/NCT04366089.

60 Pasquier JC. Efficacy of Probiotics in Reducing Duration and Symptoms of COVID-19 (PROVID-19) access 18 March 2021]. Available at: https://clinicaltrials.gov/ct2/show/ NCT04621071.

61 Desrosiers MY. Efficacy of Intranasal Probiotic Treatment to Reduce Severity of Symptoms in COVID19 Infection [last access 18 March 2021]. Available at: https://clinicaltrials.gov/ct2/show/NCT04458519,

62 Navarro L. Study to Evaluate the Effect of a Probiotic in COVID-19 [last access 18 March 2021]. Available at: https://clinicaltrials.gov/ ct2/show/NCT04390477.

63 Gea Gonzalez M. Efficacy of L. Plantarum and P. Acidilactici in Adults With SARS-CoV-2 and COVID-19 [last access 18 March 2021]. Available at: https://clinicaltrials.gov/ct2/ show/NCT04517422.

64 Stadlbauer V. Synbiotic Therapy of Gastrointestinal Symptoms During Covid-19 Infection (SynCov) access 18 March 2021]. Available at: https://clinicaltrials.gov/ct2/show/ NCT04420676.

65 Zelaya H, Alvarez S, Kitazawa H, Villena J. Respiratory antiviral immunity and immunobiotics: beneficial effects on inflammationcoagulation interaction during influenza virus infection. Front Immunol. 2016;7:633.

66 Hummel S, Veltman K, Cichon C, Sonnenborn U, Schmidt MA. Differential targeting of the E-cadherin/ $\beta$-catenin complex by grampositive probiotic lactobacilli improves epithelial barrier function. Appl Environ Microbiol. Jan 2012;78(4):1140-7.

67 d'Ettorre G, Rossi G, Scagnolari C, Andreotti M, Giustini N, Serafino S, et al. Probiotic supplementation promotes a reduction in T-cell activation, an increase in Th17 frequencies, and a recovery of intestinal epithelium integrity and mitochondrial morphology in ARTtreated HIV-1-positive patients. Immun Inflamm Dis. 2017;5(3):244-60.
68 Rishi P, Thakur K, Vij S, Rishi L, Singh A, Kaur IP, et al. Diet, gut microbiota and COVID-19. Indian J Microbiol. 2020;60(4):420-

69 Narayanan S, Pitchumoni CS. Dietary fiber. In: Pitchumoni $\mathrm{C}$, Dharmarajan T, editors. Geriatric gastroenterology. Cham: Springer; 2020. p. 1-16.

70 Senghor B, Sokhna C, Ruimy R, Lagier JC. Gut microbiota diversity according to dietary habits and geographical provenance. Hum Microbiome J. 2018;7-8:1-9.

71 De Filippo C, Di Paola M, Ramazzotti M, Albanese D, Pieraccini G, Banci E, et al. Diet, environments, and gut microbiota. A preliminary investigation in children living in rural and urban Burkina Faso and Italy. Front Microbiol. 2017;8:1979.

72 Maslowski KM, Mackay CR. Diet, gut microbiota and immune responses. Nat Immunol. 2011;12(1):5-9.d

73 Tomova A, Bukovsky I, Rembert E, Yonas W, Alwarith J, Barnard ND, et al. The effects of vegetarian and vegan diets on gut microbiota. Front Nutr. 2019;6:47.

74 World Health Organization. Coronavirus disease (COVID-19) situation report-132. 2020;31-5.

75 Marazzato M, Ceccarelli G, d'Ettorre G. Dysbiosis in SARS-CoV-2 infected patients. Gastroenterology. 2020 Dec 30. S00165085(20)35618-3.

76 Kukla M, Adrych K, Dobrowolska A, Mach T, Reguła J, Rydzewska G. Guidelines for Clostridium difficile infection in adults. Prz Gastroenterol. 2020;15(1):1-21.

77 Lamb CA, Kennedy NA, Raine T, Hendy PA, Smith PJ, Limdi JK, et al. British Society of gastroenterology consensus guidelines on the management of inflammatory bowel disease in adults. Gut. 2019 Dec;68(Suppl 3): s1-s106.

78 Landi C, Santinelli L, Bianchi L, Shaba E, Ceccarelli G, Cavallari EN, et al. Cognitive impairment and CSF proteome modification after oral bacteriotherapy in HIV patients. J Neurovirol. 2020 Feb;26(1):95-106.

79 Infusino F, Marazzato M, Mancone M, Fedele F, Mastroianni CM, Severino P, et al. Diet supplementation, probiotics, and nutraceuticals in SARS-CoV-2 infection: a scoping review. Nutrients. 2020 Jun 8;12(6):1718.

80 Vangay P, Johnson AJ, Ward TL, Al-Ghalith GA, Shields-Cutler RR, Hillmann BM, et al. US immigration westernizes the human gut microbiome. Cell. 2018;175(4):962-e10. 